\title{
Direct Cardiomyocyte Reprogramming: A New Direction for Cardiovascular Regenerative Medicine
}

\author{
B. Alexander $\mathrm{Yi}^{1}$, Christine L. Mummery ${ }^{2}$, and Kenneth R. Chien ${ }^{3,4}$ \\ ${ }^{1}$ Cardiovascular Research Center, Massachusetts General Hospital, Boston, Massachusetts 02114 \\ ${ }^{2}$ Department of Anatomy and Embryology, Leiden University Medical Center, 2300 RC Leiden, The Netherlands \\ ${ }^{3}$ Department of Stem Cell and Regenerative Biology, Harvard University, Cambridge, Massachusetts 02138 \\ ${ }^{4}$ Department of Cell and Molecular Biology and Medicine, Karolinska Institutet, SE-171 77 Stockholm, Sweden \\ Correspondence: byi@partners.org
}

The past few years have seen unexpected new developments in direct cardiomyocyte reprogramming. Direct cardiomyocyte reprogramming potentially offers an entirely novel approach to cardiovascular regenerative medicine by converting cardiac fibroblasts into functional cardiomyocytes in situ. There is much to be learned, however, about the mechanisms of direct reprogramming in order that the process can be made more efficient. Early efforts have suggested that this new technology can be technically challenging. Moreover, new methods of inducing heart reprogramming will need to be developed before this approach can be translated to the bedside. Despite this, direct cardiomyocyte reprogramming may lead to new therapeutic options for sufferers of heart disease.

$\mathrm{D}$ uring its short history, cardiovascular regenerative medicine has already ventured down a number of different paths. Because the minimal ability of cardiomyocytes to divide and proliferate had already been well documented (Soonpaa and Field 1998), the initial thrust was in identifying cells that could be delivered to the heart in the hope that they would integrate and improve cardiac function. In the 1990s, early studies examined human fetal cardiomyocytes and skeletal muscle precursors called satellite cells for this potential (Soonpaa et al. 1994; Hagege et al. 2003). Then in 2001, a study by Orlic et al. initially suggested that a subpopulation of c-kit ${ }^{+}$bone marrow-derived cells could regenerate heart tissue when transplanted into a mouse model of myocardial infarction (MI) (Orlic et al. 2001). This opened the floodgates to a number of human clinical studies and later trials testing a variety of mostly bone marrow-derived cell types in patients with both acute MI as well as chronic heart disease. These cell types include hematopoietic stem cells (HSCs), mesenchymal stem cells (MSCs), endothelial progenitor cells (EPCs), umbilical cord blood stem cells, and various adult cardiac stem cells (CSCs) (Templin et al. 2011) as well as skeletal muscle cells (Menasche 2008).

Editors: Margaret Buckingham, Christine L. Mummery, and Kenneth R. Chien

Additional Perspectives on The Biology of Heart Disease available at www.perspectivesinmedicine.org

Copyright (C) 2013 Cold Spring Harbor Laboratory Press; all rights reserved; doi: 10.1101/cshperspect.a014050

Cite this article as Cold Spring Harb Perspect Med 2013;3:a014050 
B.A. Yi et al.

Now that a number of these trials have been published including results from multiyear follow-up studies, a few broad lessons about cellbased therapy for the heart might be gleaned. Overall, the results have been inconsistent with many reporting none-to-negligible effects on the expected outcomes of a therapy aimed at cardiac regeneration with respect to improvements in left ventricular (LV) ejection fraction, fractional shortening, or infarct size (Boudoulas and Hatzopoulos 2009), whereas others have been more positive. A few, for example, have reported improvements in clinical outcomes such as the incidence of death, recurrence of MI, the need for repeat coronary revascularization, or frequency of angina (Losordo et al. 2007; Schachinger et al. 2009). Many studies, however, have not been adequately powered to evaluate the impact of cell-based therapy on clinical end points and, in light of the uncertain improvement with metrics of cardiac function, the significance of these clinical observations remains unclear.

If nothing else, though, these studies have helped bring the challenges of cardiac cell-based therapy into sharper relief. For instance, experiments studying the survival of cells injected into the cardiac environment has led to the realization that the vast number of cells do not survive because they are immunologically rejected, die from the lack of a proper cellular microenvironment, become trapped in the lung vasculature, or are passively carried away from the target region of interest via the circulation (Ptaszek et al. 2012). This has led to new innovations and experimentation in routes of delivery such as intravenous, intracoronary, intrapericardial, transepicardial, transendocardial, and coronary vein injection. But other vexing questions remain. To this day, there is a lack of consensus on what constitutes the optimal source of cells for cardiac cell therapy because there have been no head-to-head comparisons of multiple cell types, even in animal models. In addition, after delivery of cells that have been clearly predifferentiated to cardiomyocytes, results have been inconclusive on whether they will be able to properly couple and contract with existing cardiomyocytes (van Laake et al. 2007; Shiba et al. 2012; Ardehali et al. 2013). Further mud- dying waters, the field has largely moved away from the initial premise that bone marrow-derived stem cells are capable of transdifferentiating into functioning myocytes. Instead, it has become more accepted that any salutary effects of cardiac cell-based therapy occur by secreted paracrine factors or some other mechanism of ameliorating damage rather than the cells themselves (Loffredo et al. 2011; Mirotsou et al. 2011). Thus, although past studies in cell-based therapy have opened new horizons in cardiac regenerative medicine, experience has helped illustrate a number of shortcomings with this approach and the need to develop alternative approaches. Many of the shortcomings have arisen because of difficulties in accurate and unambiguous tracking of cell fate after injection.

\section{CARDIOMYOCYTE REPROGRAMMING, A NEW ROAD}

Like any organ, the heart proceeds through a series of complex steps in wound healing after injury (Blankesteijn et al. 2001). Because cardiomyocytes have a very limited capacity to divide, the final result of wound healing in the heart is the formation of a scar at the injury site that is largely comprised of fibroblasts. Although fibroblasts help maintain the structural integrity of the heart after an injury-without them, the heart after an MI would be prone to wall rupture-they have no contractile ability (Camelliti et al. 2005). This means that surviving heart myocytes have to work harder to support cardiac output. Over time, this stress triggers a downward spiral of neurohormonal activation and adverse cardiac remodeling events during which the cardiomyocytes themselves become hypertrophic, which eventually progresses to congestive heart failure (Jessup and Brozena 2003).

But what if fibroblasts in the scar could be reprogrammed to form myocytes able to beat synchronously with the rest of the heart and support cardiac pumping? This bit of "cellular alchemy" is not as surprising as it might seem. More than 20 years ago, it was discovered that expression of a single transcription factor, $M y o D$, in fibroblasts could initiate the expression of skeletal muscle genes and coax them 
into adopting the morphology of skeletal myotubes (Davis et al. 1987). Initial efforts to identify a corresponding master cardiac transcription factor that could convert fibroblasts into cardiomyocytes were discouraging (Evans et al. 1994); however, later studies suggested that a dominantly acting gene, or set of genes, that could activate the cardiomyocyte gene program may exist (Matsuura et al. 2004). Yamanaka's revolutionary discovery that a combination of four transcription factor genes could reprogram fibroblasts into induced pluripotent stem cells (Takahashi and Yamanaka 2006) and the discovery that combinations of transcription factors could induce direct reprogramming of fibroblasts to the neural lineage (Vierbuchen et al. 2010) emboldened others to try and identify a set of genes that could bypass the stem cell state and convert fibroblasts directly into beating cardiomyocytes. Given history, a search for a master cardiomyocyte regulator seemed elusive. In 2009, Takeuchi et al. showed that two wellknown cardiac transcription factors, Gata4 and $T b \times 5$, as well as a cardiac-specific subunit of the BAF chromatin remodeling complex, Baf60c, could induce the formation of ectopic beating cardiomyocytes, but this strategy seemed limited to noncardiogenic mesodermal cells in the early mouse embryo (Takeuchi and Bruneau 2009).

Therefore a report from Ieda et al. in 2010 reporting that a slightly different mix of transcription factors, Gata4, Mef2c, and Tbx5 (GMT), could rapidly and efficiently reprogram fibroblasts into functional cardiomyocytes in vitro generated much interest (Ieda et al. 2010). They had isolated neonatal cardiac fibroblasts from a mouse strain in which the promoter for the $\alpha$-myosin heavy chain $(\alpha-\mathrm{MHC})$ gene could drive the expression of green fluorescent protein (GFP) and then tested a pool of 14 known cardiogenic transcription factors by retroviral transduction for their ability to generate $\alpha$-MHC-GFP ${ }^{+}$cells by fluorescence-activated cell sorting (FACS). They must have known they were on the right track when with all 14 genes they were able to detect a small percentage of $\mathrm{GFP}^{+}$cells. From there, they underwent a strategy of single factor elimination before narrowing the factors to GMT. They gave the $\alpha$ -

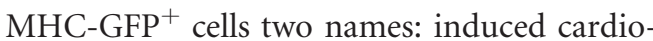
myocytes (iCMs) and cardiomyocyte-like cells.

However, follow-up efforts at direct cardiomyocyte reprogramming raised a number of questions. For instance, although Ieda and colleagues (leda et al. 2010) showed that GMT expression in cardiac fibroblasts could readily generate a population of $\alpha$-MHC-GFP ${ }^{+}$cells within days, only a fraction of cells in that population also expressed cardiac troponin $\mathrm{T}$ (cTnT) and an even smaller fraction of those cells went on to beat spontaneously after 4-5 weeks of culture. Moreover, Ieda and colleagues reported that they observed no beating at all when they expressed GMT in tail-tip fibroblasts (Srivastava and Ieda 2012). These observations stood in stark contrast to the spontaneously beating cardiomyocytes that are readily seen at high frequency upon differentiation of embryonic stem cells and induced pluripotent stem cells (Mummery et al. 2003; Narazaki et al. 2008), and undercut their claims that direct cardiomyocyte reprogramming was a rapid and efficient technique of generating functionally beating cardiomyocytes. Other groups have since reported that they see no spontaneous beating at all when using lentiviruses to express GMT in cardiac fibroblasts (Chen et al. 2012), leaving open the possibility that the rare occurrences of beating cardiomyocytes observed in the original study could have arisen from contaminating immature cardiomyocytes or cardiovascular progenitors in the starting neonatal cardiac fibroblast population (Protze et al. 2012). Indeed, others have found that in isolating populations of cardiac fibroblasts, there is a very small, but nonzero, population of contaminating cardiomyocytes or $\mathrm{c}-\mathrm{kit}^{+} / \mathrm{sca}^{-}{ }^{+}$cardiovascular progenitor cells even after FACS sorting or Percoll gradient centrifugation that increases in number over time (Jayawardena et al. 2012; Protze et al. 2012).

Another question has been: How close are cardiomyocyte-like cells to bona fide functioning cardiomyocytes? Despite the global gene expression analysis that clustered induced cardiomyocytes closer to neonatal cardiomyocytes than cardiac fibroblasts (Ieda et al. 2010), other reports have since shown that GMT is margin- 
B.A. Yi et al.

ally effective at activating the full cardiomyocyte gene program (Chen et al. 2012). For instance, Protze and colleagues (Protze et al. 2012) have shown that GMT can turn on expression of $\alpha$ MHC as well as cTnT; however, that combination was less effective at activating expression of myosin regulatory light chain 2 ( $M y l 2$, also known as MLC2v), the cardiac homeobox transcription factor $(N k \times 2.5)$, or the voltage-gated sodium channel $\mathrm{Na}_{\mathrm{v}} 1.5$ (Scn5a). Thus, in retrospect, Ieda's use of a single gene reporter readout, $\alpha$-MHC, to screen for factors that reprogram fibroblasts into cardiomyocytes may have been serendipitous, and had they chosen another reporter, their initial experiments might not have yielded anything at all. This also suggests that another possibility for why there was such a low percentage of spontaneous beating in their induced cardiomyocytes is because those cells were incompletely reprogrammed to the cardiomyocyte state and that the addition of other factors or culture conditions might improve the rate or efficiency of cardiomyocyte reprogramming (Srivastava and Ieda 2012). Indeed, others have reported success with slightly modified combinations such as Mef2c, Tbx5, and myocardin (MT-Myocd) (Protze et al. 2012) or GMT plus Hand2 (GHMT) (Song et al. 2012). Jayawardena et al. (2012) reported direct cardiomyocyte reprogramming using microRNAs, small bits of RNA that are capable of binding to target messenger RNAs and targeting them for degradation. They reported that a single transient transfection of microRNAs miR-1 alone or in combination with miR-133, miR208, or miR-499 can activate molecular pathways in cardiac fibroblasts and induce the formation of sarcomere-like structures and spontaneous calcium fluxes and cell contractions.

Even with the caveats described here, however, it appears probable that GMT or other sets of cardiogenic factors can indeed activate the expression of cardiomyocyte genes that over time may induce cells to adopt a cardiomyocyte-like cell fate. For this transdifferentiation to occur, the cells would have had to have undergone dramatic changes in their epigenetic state to promote the durable expression of cardiomyocyte proteins to support the formation of the unique phenotypic features of cardiac myocytes such as sarcomeres as well as electrophysiological action potentials (Chien et al. 2012). Thus far, it appears reasonable to conclude that inducing fibroblasts to fully traverse this epigenetic landscape to the fully reprogrammed cardiomyocyte state in vitro is technically challenging, relatively inefficient, or both (Srivastava and Ieda 2012).

\section{GOING IN VIVO}

Despite these challenges, researchers pressed forward by testing direct cardiomyocyte reprogramming in vivo in the hopes that the cardiac microenvironment might promote the fibroblasts-to-cardiomyocyte reprogramming. In two scientific studies published in 2012, the Srivastava group and the Olson group showed that cardiomyocyte reprogramming is indeed more efficient in vivo (Fig. 1). Moreover, their studies suggested that delivery of direct reprogramming factors into the wound healing environment of the heart in a mouse MI model can lead to functional improvements in heart function that persist even months later (Qian et al. 2012; Song et al. 2012). Qian et al. used retroviruses to deliver GMT into the mouse heart. To keep track of cardiac fibroblasts that had been reprogrammed into cardiomyocytes and preexisting cardiomyocytes, they did their experiments in a mouse strain in which cardiac fibroblasts were genetically labeled with $\beta$-galactosidase $(\beta$-gal) by driving its expression from a promoter for genes associated with cardiac fibroblasts such as periostin or fibroblast-specific protein (Fsp1) (Qian et al. 2012; Song et al. 2012). Because retroviruses can only stably integrate into the genomes of actively dividing cells, this approach helped to direct the reprogramming factors to cardiac fibroblasts. It turned out that this strategy was markedly effective at preventing any stray retroviruses from infecting cardiomyocytes. After surgical ligation of the coronary artery and injection of retroviruses, they saw numerous numbers of $\beta-$ gal $^{+} / \alpha$-actinin ${ }^{+}$cells in the infarct zones but practically zero numbers of $\beta$ gal cardiomyocytes that would suggest spurious activation of these genetic markers in cardio- 


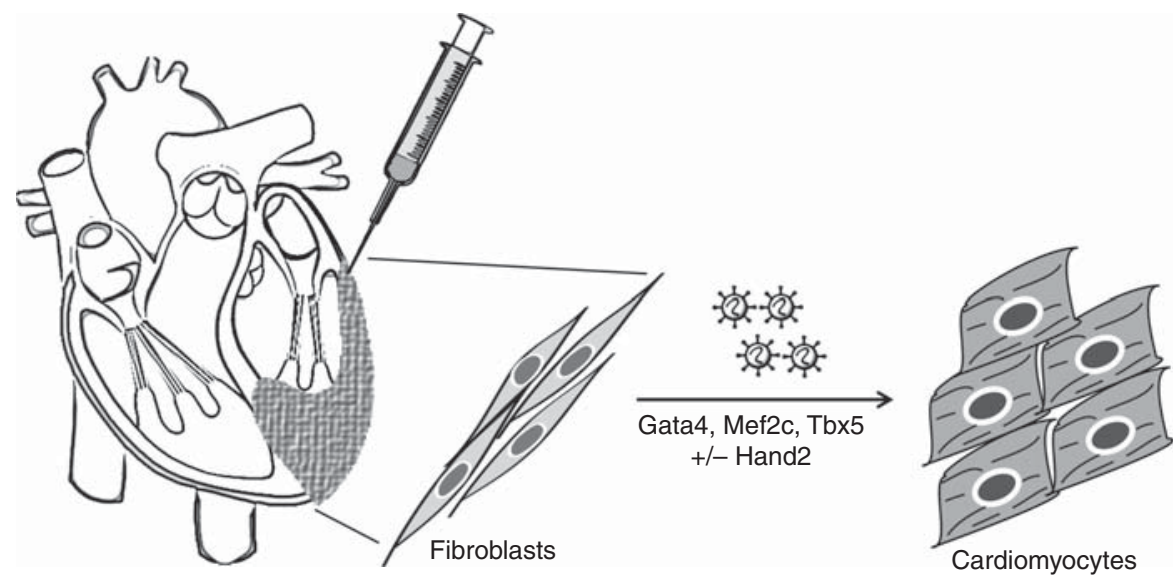

Figure 1. Direct cardiomyocyte reprogramming offers a potentially novel approach to cardiovascular regenerative medicine. In a mouse model of myocardial infarction, retroviral particles for cardiogenic factors directly injected into injured regions of the mouse heart (stippled area) may be used to restore cardiac function by reprogramming fibroblasts to form beating cardiomyocytes. Qian et al. (2012) and Inagawa et al. (2012) both used the cardiac transcription factors Gata4, Mef2c, and Tbx5, whereas Song et al. (2012) included Hand2.

myocytes or evidence of these markers being activated in bone marrow-derived cells. This finding greatly simplified the interpretation of their studies.

Because cardiac wound healing is a complex milieu of inflammatory cells that migrate in, break down necrotic cells, and resorb cellular debris to assist in scar formation (Blankesteijn et al. 2001), Qian et al. also wanted to rule out the possibility that the $\beta$-gal ${ }^{+} / \alpha$-actinin ${ }^{+}$cells they observed arose from unusual cell fusion events between cardiac fibroblasts and damaged cardiomyocytes. They did this using another transgenic mouse line in which cardiomyocytes could be irreversibility labeled with yellow fluorescent protein (Myh6-merCremer:R26R enhanced yellow fluorescent protein [EYFP]). After pulse labeling cardiomyocytes with yellow fluorescent protein (YFP), they redid their experiment. Four weeks after inducing an MI and delivering the retroviruses to transduce GMT and dsRed to label the infected cells, they observed no $\mathrm{YFP}^{+} / \mathrm{dsRed}^{+}$double positive cardiomyocytes suggesting that no new cardiomyocytes had arisen as a result of fusion between cardiac fibroblasts infected with dsRed and $\mathrm{YFP}^{+}$cardiomyocytes. Indeed, this experiment helped confirm their findings that GMT was efficient at reprogramming fibroblasts into cardiomyocytes in vivo. In their analysis, they were able to discern a decrease in the percentage of $\mathrm{YFP}^{+}$cardiomyocytes, implying that new unlabeled cardiomyocytes that had originated from fibroblasts had diluted their relative numbers. In what had to be a delicate experiment, they were further able to dissect a $1 \mathrm{~mm}$ border zone around the infarcts they created and quantified numbers of $\mathrm{YFP}^{+}$cells in the border zone. Strikingly, they report that $\sim 35 \%$ of the cardiomyocytes in the border zone were newly generated induced cardiomyocytes, which represents a marked improvement in the reprogramming efficiency over their in vitro results (Ieda et al. 2010). This figure, however, may not reflect all fully reprogrammed cells. An examination of reprogrammed induced cardiomyocytes from heart sections revealed a continuum of reprogrammed cells with morphological and functional features that ranged from partially reprogrammed cells to mature cardiomyocytes with almost half of these new myocytes showing well-organized sarcomeres after 4 weeks. Moreover, they observed examples of new cardiomyocytes that had coupled to surrounding car- 
B.A. Yi et al.

diomyocytes with intercalated disks and gap junctions that stain for $\mathrm{N}$-cadherin or connexin-43 and they showed that the gap junctions were functional by microinjecting dyes and demonstrating intercellular transfer between cardiomyocytes (Qian et al. 2012). In sum, these results represent a marked advance over cardiac cell-based therapy in which cellcardiomyocyte coupling, much less cell survival, has remained a significant issue.

The question remains whether these new cardiomyocytes make any difference to cardiac function. To address this, the groups performed mini-mouse clinical trials (Qian et al. 2012; Song et al. 2012). They did these experiments in a blinded fashion so that the researchers measuring cardiac function did not know which mice had received the retroviral infections containing GMT or GHMT and which had received a control. At different time points after the surgery, they evaluated mouse cardiac function using echocardiography or magnetic resonance imaging. Both modalities showed unequivocally that delivering reprogramming factors retrovirally to the mouse heart improved cardiac parameters such as fractional shortening, stroke volume, and cardiac output. Song et al. reported that 6 weeks after the surgery, mice that had received GHMT had almost twice the improvement in ejection fraction compared with control mice (49\% in mice receiving GHMT versus $28 \%$ in controls compared with $>70 \%$ in unoperated mice) and these improvements persisted even at 12 weeks after the procedure (Song et al. 2012). Moreover, histologically, the region where the retrovirus was delivered also showed other potentially beneficial findings such as increased capillary density (Qian et al. 2012). Importantly, Qian and colleagues detected no arrhythmias in the mice who had received the reprogramming factors, perhaps reflecting the cell-cell coupling they had observed between new cardiomyocytes and existing cardiomyocytes (Qian et al. 2012). Although it is tempting to suggest that the near normalization of cardiac function they report is owing to the active contractions of newly reprogrammed cardiomyocytes, the mechanism of these improvements in mouse remains unclear and a primary role of paracrine factors in the heart cannot be ruled out (Qian et al. 2012; Song et al. 2012). The numbers of new cardiomyocytes were too low to be a likely explanation for all of the improvement detected in cardiac function.

With regard to these studies, several points warrant mention. First, mindful of the technical challenges with in vitro cardiomyocyte reprogramming, it may be prudent to wait until others have had an opportunity to reproduce these results with GMT, GHMT, or the other combinations reported. Already, a separate group led by one of the original investigators of in vitro reprogramming has published their own experience with direct cardiomyocyte reprogramming in vivo (Inagawa et al. 2012). Interestingly, using retroviral expression of GFP to test in vivo infection, they observed that GFP expression was readily observable in the first week but thereafter became reduced at 2 weeks and was barely detectable after 4 weeks. They hypothesized that this attenuation of expression might be owing to an immune response because retroviral gene expression was more persistent when they did their experiments in the immunosuppressed nude mouse. But even in nude mice, they report much less efficient cardiomyocyte reprogramming $(\sim 1 \%)$ in their mouse $\mathrm{MI}$ experiments with GMT. Perhaps as a by-product of their reduced expression levels, even after 1 month, they report that the induced cardiomyocytes in vivo appeared smaller than endogenous ventricular myocytes and a much lower percentage of cells $(\sim 15 \%)$ showed clear sarcomere-like striations (Inagawa et al. 2012). Overall, they report their reprogramming efficiency to be 10-fold less than that reported in Qian et al., whereas the efficiency reported by Song et al. with GHMT ( $6.5 \%)$ appears to be somewhere in the middle (Inagawa et al. 2012). Moreover, Inagawa et al. report that they also attempted to use mouse transgenic lines to specifically label cardiac fibroblasts in their experiments similar to those experiments reported in the previous studies, but they found that their fibroblast-lineage tracing mice were not suitable because of leakiness in reporter expression (Inagawa et al. 2012). 


\section{OF MICE AND MEN}

Despite these interesting preclinical studies in mice, there are a number of hurdles that must be overcome before direct cardiomyocyte reprogramming can be studied in human clinical trials. One is that it is not feasible to introduce retrovirus as therapy in humans and hence it will be necessary to induce reprogramming genes using other delivery means perhaps by adeno-associated viral vectors, chemically modified RNA, or small molecules (Srivastava and Ieda 2012). Another challenge is that human fibroblasts appear to be more difficult to reprogram than mouse fibroblasts. Recently, the Olson laboratory reported that they were able to achieve direct cardiomyocyte reprogramming in human fibroblasts (Nam et al. 2013). Starting from human dermal fibroblasts, they were initially unsuccessful using GHMT, but based on prior understanding of cardiomyocyte gene networks, they were successful by including myocardin as well as two microRNAs, miR-1 and miR-133, with GHMT and waiting 4-11 weeks for fibroblasts to form sarcomere-like structures, calcium transients, and finally show spontaneous contractions.

Although the past few years have seen significant advances in cardiomyocyte direct reprogramming, there is also an urgent need to replicate these studies and to better understand its mechanism in the hopes that this technology can be made more efficient before being translated to the bedside. It seems safe to conclude that direct cardiomyocyte reprogramming is slow, inefficient, and technically challenging. As if to underline the point, the Olson laboratory in their report of human cardiomyocyte reprogramming state that they were unable to activate expression of cardiac markers in mouse fibroblasts using the microRNAs reported by Jayawardena and colleagues (Nam et al. 2013). The wide variability in published reports of in vitro and in vivo reprogramming might reflect the need to use high titers of viruses as well as achieve a precise stoichiometry of reprogramming factors in fibroblasts that are epigenetically receptive to reprogramming. Nevertheless, this approach offers some optimism for sufferers of heart disease. With the myriad of new approaches to cardiovascular regenerative medicine that have been studied in recent years, one of them may ultimately have the potential to transform cardiovascular care in the 21 st century by reducing mortality and improving the lives of patients with heart disease.

\section{ACKNOWLEDGMENTS}

The authors thank Emil Hansson for thoughtful comments on the manuscript. B.A.Y. is supported by an award from the American Heart Association (12FTF11820037) and the Harvard Stem Cell Institute. C.L.M. is supported by the CVON-HUSTCARE Programme of the Netherlands Heart Foundation.

\section{REFERENCES}

Ardehali R, Ali SR, Inlay MA, Abilez OJ, Chen MQ, Blauwkamp TA, Yazawa M, Gong Y, Nusse R, Drukker M, et al. 2013. Prospective isolation of human embryonic stem cell-derived cardiovascular progenitors that integrate into human fetal heart tissue. Proc Natl Acad Sci 110: 3405-3410.

Blankesteijn WM, Creemers E, Lutgens E, Cleutjens JP, Daemen MJ, Smits JF. 2001. Dynamics of cardiac wound healing following myocardial infarction: Observations in genetically altered mice. Acta Physiol Scand 173: $75-82$.

Boudoulas KD, Hatzopoulos AK. 2009. Cardiac repair and regeneration: The Rubik's cube of cell therapy for heart disease. Dis Model Mech 2: 344-358.

Camelliti P, Borg TK, Kohl P. 2005. Structural and functional characterisation of cardiac fibroblasts. Cardiovasc Res 65: $40-51$.

Chen JX, Krane M, Deutsch MA, Wang L, Rav-Acha M, Gregoire S, Engels MC, Rajarajan K, Karra R, Abel ED, et al. 2012. Inefficient reprogramming of fibroblasts into cardiomyocytes using Gata4, Mef2c, and Tbx5. Circ Res 111: $50-55$.

Chien KR, Yi BA, Xu H, Mummery CL. 2012. Cardiomyocyte reprogramming and the new age of cellular alchemy. J Mol Cell Cardiol 53: 311-313.

Davis RL, Weintraub H, Lassar AB. 1987. Expression of a single transfected cDNA converts fibroblasts to myoblasts. Cell 51: 987-1000.

Evans SM, Tai LJ, Tan VP, Newton CB, Chien KR. 1994. Heterokaryons of cardiac myocytes and fibroblasts reveal the lack of dominance of the cardiac muscle phenotype. Mol Cell Biol 14: 4269-4279.

Hagege AA, Carrion C, Menasche P, Vilquin JT, Duboc D, Marolleau JP, Desnos M, Bruneval P. 2003. Viability and differentiation of autologous skeletal myoblast grafts in ischaemic cardiomyopathy. Lancet 361: 491-492. 
B.A. Yi et al.

Ieda M, Fu JD, Delgado-Olguin P, Vedantham V, Hayashi Y, Bruneau BG, Srivastava D. 2010. Direct reprogramming of fibroblasts into functional cardiomyocytes by defined factors. Cell 142: 375-386.

Inagawa K, Miyamoto K, Yamakawa $\mathrm{H}$, Muraoka N, Sadahiro T, Umei T, Wada R, Katsumata Y, Kaneda R, Nakade $\mathrm{K}$, et al. 2012. Induction of cardiomyocyte-like cells in infarct hearts by gene transfer of Gata4, Mef2c, and Tbx5. Circ Res 111: 1147-1156.

Jayawardena TM, Egemnazarov B, Finch EA, Zhang L, Payne IA, Pandya K, Zhang Z, Rosenberg P, Mirotsou M, Dzau VJ. 2012. MicroRNA-mediated in vitro and in vivo direct reprogramming of cardiac fibroblasts to cardiomyocytes. Circ Res 110: 1465-1473.

Jessup M, Brozena S. 2003. Heart failure. N Engl J Med 348: 2007-2018.

Loffredo FS, Steinhauser ML, Gannon J, Lee RT. 2011. Bone marrow-derived cell therapy stimulates endogenous cardiomyocyte progenitors and promotes cardiac repair. Cell Stem Cell 8: 389-398.

Losordo DW, Schatz RA, White CJ, Udelson JE, Veereshwarayya V, Durgin M, Poh KK, Weinstein R, Kearney M, Chaudhry M, et al. 2007. Intramyocardial transplantation of autologous $\mathrm{CD} 34^{+}$stem cells for intractable angina: A phase I/IIa double-blind, randomized controlled trial. Circulation 115: 3165-3172.

Matsuura K, Wada H, Nagai T, Iijima Y, Minamino T, Sano M, Akazawa H, Molkentin JD, Kasanuki H, Komuro I. 2004. Cardiomyocytes fuse with surrounding noncardiomyocytes and reenter the cell cycle. J Cell Biol 167: 351363.

Menasche P. 2008. Skeletal myoblasts and cardiac repair. J Mol Cell Cardiol 45: 545-553.

Mirotsou M, Jayawardena TM, Schmeckpeper J, Gnecchi M, Dzau VJ. 2011. Paracrine mechanisms of stem cell reparative and regenerative actions in the heart. $J$ Mol Cell Cardiol 50: 280-289.

Mummery C, Ward-van Oostwaard D, Doevendans P, Spijker R, van den Brink S, Hassink R, van der Heyden M, Opthof T, Pera M, de la Riviere AB, et al. 2003. Differentiation of human embryonic stem cells to cardiomyocytes: Role of coculture with visceral endodermlike cells. Circulation 107: 2733-2740.

Nam YJ, Song K, Luo X, Daniel E, Lambeth K, West K, Hill JA, Dimaio JM, Baker LA, Bassel-Duby R, et al. 2013. Reprogramming of human fibroblasts toward a cardiac fate. Proc Natl Acad Sci 110: 5588-5593.

Narazaki G, Uosaki H, Teranishi M, Okita K, Kim B, Matsuoka S, Yamanaka S, Yamashita JK. 2008. Directed and systematic differentiation of cardiovascular cells from mouse induced pluripotent stem cells. Circulation 118: 498-506.

Orlic D, Kajstura J, Chimenti S, Jakoniuk I, Anderson SM, Li B, Pickel J, McKay R, Nadal-Ginard B, Bodine DM, et al. 2001. Bone marrow cells regenerate infarcted myocardium. Nature 410: 701-705.
Protze S, Khattak S, Poulet C, Lindemann D, Tanaka E, Ravens U. 2012. A new approach to transcription factor screening for reprogramming of fibroblasts to cardiomyocyte-like cells. J Mol Cell Cardiol 53: 323-332.

Ptaszek LM, Mansour M, Ruskin JN, Chien KR. 2012. Towards regenerative therapy for cardiac disease. Lancet 379: 933-942.

Qian L, Huang Y, Spencer CI, Foley A, Vedantham V, Liu L, Conway SJ, Fu JD, Srivastava D. 2012. In vivo reprogramming of murine cardiac fibroblasts into induced cardiomyocytes. Nature 485: 593-598.

Schachinger V, Assmus B, Erbs S, Elsasser A, Haberbosch W, Hambrecht R, Yu J, Corti R, Mathey DG, Hamm CW, et al. 2009. Intracoronary infusion of bone marrow-derived mononuclear cells abrogates adverse left ventricular remodelling post-acute myocardial infarction: Insights from the reinfusion of enriched progenitor cells and infarct remodelling in acute myocardial infarction (REPAIR-AMI) trial. Eur J Heart Fail 11: 973-979.

Shiba Y, Fernandes S, Zhu WZ, Filice D, Muskheli V, Kim J, Palpant NJ, Gantz J, Moyes KW, Reinecke H, et al. 2012. Human ES-cell-derived cardiomyocytes electrically couple and suppress arrhythmias in injured hearts. Nature 489: $322-325$.

Song K, Nam YJ, Luo X, Qi X, Tan W, Huang GN, Acharya A, Smith CL, Tallquist MD, Neilson EG, et al. 2012. Heart repair by reprogramming non-myocytes with cardiac transcription factors. Nature 485: 599-604.

Soonpaa MH, Field LJ. 1998. Survey of studies examining mammalian cardiomyocyte DNA synthesis. Circ Res 83: $15-26$.

Soonpaa MH, Koh GY, Klug MG, Field LJ. 1994. Formation of nascent intercalated disks between grafted fetal cardiomyocytes and host myocardium. Science 264: 98-101.

Srivastava D, Ieda M. 2012. Critical factors for cardiac reprogramming. Circ Res 111: 5-8.

Takahashi K, Yamanaka S. 2006. Induction of pluripotent stem cells from mouse embryonic and adult fibroblast cultures by defined factors. Cell 126: 663-676.

Takeuchi JK, Bruneau BG. 2009. Directed transdifferentiation of mouse mesoderm to heart tissue by defined factors. Nature 459: 708-711.

Templin C, Luscher TF, Landmesser U. 2011. Cell-based cardiovascular repair and regeneration in acute myocardial infarction and chronic ischemic cardiomyopathycurrent status and future developments. Int J Dev Biol 55: 407-417.

van Laake LW, Passier R, Monshouwer-Kloots J, Verkleij AJ, Lips DJ, Freund C, den Ouden K, Ward-van Oostwaard D, Korving J, Tertoolen LG, et al. 2007. Human embryonic stem cell-derived cardiomyocytes survive and mature in the mouse heart and transiently improve function after myocardial infarction. Stem Cell Res 1: 9-24.

Vierbuchen T, Ostermeier A, Pang ZP, Kokubu Y, Sudhof TC, Wernig M. 2010. Direct conversion of fibroblasts to functional neurons by defined factors. Nature 463: 10351041 . 


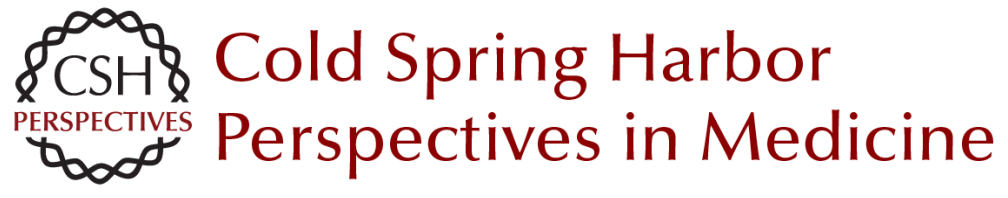

\title{
Direct Cardiomyocyte Reprogramming: A New Direction for Cardiovascular Regenerative Medicine
}

\author{
B. Alexander Yi, Christine L. Mummery and Kenneth R. Chien
}

Cold Spring Harb Perspect Med 2013; doi: 10.1101/cshperspect.a014050

\section{Subject Collection The Biology of Heart Disease}

The Genetic Basis of Aortic Aneurysm

Mark E. Lindsay and Harry C. Dietz

\section{Personalized Genomes and Cardiovascular Disease \\ Kiran Musunuru}

Complex Genetics and the Etiology of Human Congenital Heart Disease

Bruce D. Gelb and Wendy K. Chung

Genetic Networks Governing Heart Development Ashley J. Waardenberg, Mirana Ramialison, Romaric Bouveret, et al.

Heart Fields and Cardiac Morphogenesis Robert G. Kelly, Margaret E. Buckingham and Antoon F. Moorman

Regenerative Medicine: Transforming the Drug Discovery and Development Paradigm Sotirios K. Karathanasis

Myocardial Tissue Engineering: In Vitro Models Gordana Vunjak Novakovic, Thomas Eschenhagen and Christine Mummery

Pluripotent Stem Cell Models of Human Heart

Disease

Alessandra Moretti, Karl-Ludwig Laugwitz, Tatjana Dorn, et al.
Cardiac Cell Lineages that Form the Heart Sigolène M. Meilhac, Fabienne Lescroart, Cédric Blanpain, et al.

Synthetic Chemically Modified mRNA (modRNA):

Toward a New Technology Platform for

Cardiovascular Biology and Medicine

Kenneth R. Chien, Lior Zangi and Kathy O. Lui

Next-Generation Models of Human Cardiogenesis via Genome Editing Xiaojun Lian, Jiejia Xu, Jinsong Li, et al.

How to Make a Heart Valve: From Embryonic Development to Bioengineering of Living Valve

Substitutes

Donal MacGrogan, Guillermo Luxán, Anita Driessen-Mol, et al.

Insights into the Genetic Structure of Congenital Heart Disease from Human and Murine Studies on Monogenic Disorders

Terence Prendiville, Patrick Y. Jay and William T. $\mathrm{Pu}$

Cardiovascular Drug Discovery: A Perspective from a Research-Based Pharmaceutical Company G. Gromo, J. Mann and J.D. Fitzgerald

Genetics and Disease of Ventricular Muscle Diane Fatkin, Christine E. Seidman and Jonathan G. Seidman

Embryonic Heart Progenitors and Cardiogenesis Thomas Brade, Luna S. Pane, Alessandra Moretti, et al.

For additional articles in this collection, see http://perspectivesinmedicine.cshlp.org/cgi/collection/ 